\title{
Extrachromosomal HPV-16 LCR transcriptional activation by HDACi opposed by cellular differentiation and DNA integration
}

\author{
Ekaterina Dimitrova Bojilova ${ }^{1}$, Christine Weyn ${ }^{1}$, Marie-Hélène Antoine ${ }^{2}$, Véronique \\ Fontaine $^{1}$ \\ 1Université Libre de Bruxelles (ULB), Faculty of Pharmacy, Unit of Pharmaceutical Microbiology and Hygiene, 1050 Brussels, \\ Belgium \\ ${ }^{2}$ Université Libre de Bruxelles (ULB) Faculty of Medicine, Laboratory of Experimental Hormonology, 1070 Brussels, Belgium \\ Correspondence to: Véronique Fontaine, email: vfontain@ulb.ac.be \\ Keywords: human papillomavirus, histone deacetylase inhibitor, transcription, integration, differentiation \\ Received: April 07, $2016 \quad$ Accepted: September 13, $2016 \quad$ Published: September 26, 2016
}

\section{ABSTRACT}

Histone deacetylase inhibitors (HDACi) have been shown to render HPVcarrying cells susceptible to intrinsic and extrinsic apoptotic signals. As such, these epigenetic drugs have entered clinical trials in the effort to treat cervical cancer. Here, we studied the effect of common HDACi, with an emphasis on Trichostatin A (TSA), on the transcriptional activity of the HPV-16 Long Control Region (LCR) in order to better understand the impact of these agents in the context of the HPV life cycle and infection. HDACi strongly induced transcription of the firefly luciferase reporter gene under the control of the HPV-16 LCR in a variety of cell lines. In the HaCaT keratinocyte cell line undergoing differentiation induced by TSA, we observed a reduction in LCR-controlled transcription. Three major AP-1 binding sites in the HPV-16 LCR are involved in the regulation by TSA. However, whatever the status of differentiation of the HaCaT cells, TSA induced integration of extra-chromosomal transfected DNA into the cellular genome. Although these data suggest caution using HDACi in the treatment of HR HPV infection, further in vivo studies are necessary to better assess the risk.

\section{INTRODUCTION}

More than 120 human papillomaviruses (HPV) have been described and shown to be globally distributed. They seem to coexist with their host over a long period, sometimes in a latent life cycle, as suggested by the wide variety of different types detected at random sites of healthy skin $[1,2]$. Persistent genital infections by some specific types of the alpha-papillomavirus genus, called high-risk HPV (HR-HPV), have been associated with a high risk of cervical malignant progression, most notably HPV type 16 (HPV-16) [3].

Studies of the ecto-endocervical junctions revealed residual embryonic cell populations able to differentiate and characterized by vulnerability to undergo neoplastic transformation [4]. Mirkovic et al. suggest that these cells, when infected by HR HPV, might comprise a nidus for early lesion development [5]. Following viral entry into such cells, the viral particle is uncoated, DNA is trafficked to the nucleus where viral episomal replication can occur, replicating HPV genomes to about a 100 episomal copies per cell [6]. Integration of the viral genome into the host DNA can take place, especially in case of long-term maintenance in the basal epithelium [3].

Viral episomes are retained in the nucleus and coordinately partitioned into daughter cells during mitotic division. The ability to maintain a stable copy number is an attribute of all HPVs, and has been thought to require early viral proteins such as the oncogenic E6 and E7 [7, 8], the $\mathrm{E} 1$ helicase and the $\mathrm{E} 2$ protein binding the mitotic chromosome-associated Brd4 protein $[9,10]$.

Reversible acetylation of histones is a crucial event in the regulation of gene expression [11]. This process is orchestrated by histone acetyltransferases (HATs) and histone deacetylases (HDACs). Acetylation at particular lysine residues on histones is associated with a relaxed, open chromatin configuration and therefore increased accessibility of transcription factors to their binding 
sites on target genes. Conversely, deacetylation of these lysines by HDACs leads to a more condensed chromatin structure around promoters and cis-elements, and therefore - to silencing of transcription. Thus, inhibitors of HDACs (HDACi) perturb the cellular transcription environment to a significant extent. HDACi have been shown to alter the expression of about $2 \%$ of cellular genes, including genes controlling the cell cycle and apoptosis, such as p21(waf1), c-myc, and p53 [12-15]. A study by Choi et $a l$. has shown that acetylation of histone $\mathrm{H} 4$ is markedly increased after treatment with Trichostatin A (TSA), the most potent HDACi known to date [16].

Several studies have shown that HDACi induce apoptosis in HPV-positive cell lines [17, 18] and arrest their growth independently of E6/E7 oncogene expression $[19,20]$. Consequently, HDACi could be attractive drugs for the treatment of HPV infection and entered clinical trials. On the other hand, HDACi are also well-known to activate many viral promoters, such as that of HIV-1, neurotropic JC polyomavirus, Epstein-Barr virus (EBV), Kaposi virus and even HPV-11 [21-25]. As such, they have emerged as potential drugs to, for example, "shock and kill" latent HIV-1 reservoirs not susceptible to combinatory antiretroviral therapy (CART) [22, 26-29].

Although the chromatin structure of the intrachromosomal HPV-16 LCR has been reported to repress its transcriptional activity [30], little is known on the impact of HDACi on episomal HPV-16 LCR [31]. As HPV infections and multiple co-infections are common and ubiquitous, we investigated whether HDACi can also enhance HPV-16 long-control-region (LCR)-induced transcription and the impact of HDACi on the integration of extra-chromosomal DNA into host DNA.

\section{RESULTS}

\section{Histone Deacetylase Inhibitors (HDACi) induce the HPV-16 Long Control Region (LCR) transcriptional activity in transformed human cell lines}

We investigated the capacity of the HDACi Valproate (VPA), sodium butyrate (NaBut) and Trichostatin A (TSA) to modulate induction of the luciferase reporter gene expression under the control of the HPV-16 LCR. Transient transfections were performed in three different immortalized human cell lines: BeWo (trophoblastic cell line), HeLa (cervical cell line) and $\mathrm{SiHa}$ (cervical cell line). As shown in Figure 1, all three HDACi increased the luciferase activity, not only in HPV-positive cell lines $\mathrm{SiHa}$ and HeLa, but also in the HPV-negative cell line BeWo (Figure 1). We next tested a keratinocyte cell line, $\mathrm{HaCaT}$ - a spontaneously-transformed epithelial cell line which does not contain HPV DNA. We chose to use the HDAC inhibitors VPA and TSA, as they had given the strongest induction of the HPV-16 LCR in our initial experiments (Figure 1A, 1B, 1C) and were less toxic to $\mathrm{HaCaT}$ cells than $\mathrm{NaBut}$. As seen from the last panel in Figure 1D, VPA and TSA induced several-fold (2-4 fold) the luciferase activity, compared to non-induced control (NI).

\section{The effect on HPV-16 LCR induction by the HDACi TSA is time-dependent in $\mathrm{HaCaT}$ cells}

The data presented so far could indicate an involvement of HDACi in LCR-regulated transcriptional activity. However, these data could also reflect the involvement of HDACi in other host cell modifications, such as differentiation, or additional viral mechanisms but it could also reflect a potential effect on the transfection efficiency. To assess the latter, we compared the impact of adding TSA either at the time of transfection, "T0", as previously done (Figure 1), with potentially more impact on transfection efficiency, or six hours post-transfection, "T6", with potentially less impact on transfection efficiency, as the establishment of DNA into cells after transfection generally occurs 4-6 hours post-transfection (ViaFect technical manual). We decided to use TSA as a representative $\mathrm{HDACi}$ as it had the strongest inductive effect on the expression of the luciferase gene compared to the other inhibitors, VPA and NaBut (Figure 1). As shown in Figure 2A and 2B, the effect of TSA was comparable at $\mathrm{T} 6$ and $\mathrm{T} 0$ in HeLa and $\mathrm{SiHa}$ cells, increasing strongly the LCR transcriptional activity. This suggested that the activation of the LCR transcriptional response by TSA didn't result from a potential bias of TSA on the transfection efficiency. In HaCaT cells, TSA also strongly increased the LCR transcriptional activity at T0. However, at T6, the drug unexpectedly inhibited in a dose-dependent manner the activity of the HPV-16 LCR (Figure 2B, lower panel). It is worth noting that, in our transfection controls, we could also not detect an effect of the TSA treatment (at T0 or T6) on the transfection efficiency, comparing the pCMV-eGFP transfected HaCaT untreated or TSA treated cells (T0 and T6) by visual inspection under the fluorescence microscope (data not shown).

\section{Time-dependent effect of TSA on HPV-16 LCR- driven luciferase expression in $\mathrm{HaCaT}$ cells is independent of viral early gene expression}

In order to investigate whether the presence of early proteins in HaCaT cells could suppress the HPV-16 LCR transcriptional inhibition induced by TSA $6 \mathrm{~h}$ posttransfection, we expressed all the HPV-16 early proteins under the control of their own promoter, the HPV-16 LCR, in our bioassay system (plasmid pLCRearly). We co-transfected cells with pWtLCRluc (allowing for read-out) and pLCRearly in a 2:1 ratio, and treated the transfected cells with increasing doses of TSA at the time 
of transfection (T0) or post-transfection (T6). Although HPV-16 early proteins expression strongly induce the LCR transcriptional activity in all the cells (Figure 3), the TSA time-dependent response in $\mathrm{HaCaT}$ cells was not influenced by HPV-16 early genes expression.

\section{TSA treatment of $\mathrm{HaCaT}$ cells post-transfection increases integration of the plasmid DNA into the host genome}

The TSA time-dependent response in $\mathrm{HaCaT}$ cells suggested a possible effect of TSA on the persistence of the transfected DNA. The impact of TSA treatment on DNA integration in terms of stable transfection efficiency was investigated for the first time. As shown in Figure 4A and 4B, TSA increased the number of G418-resistant clones. The effect was even more pronounced when cells were treated with TSA six hours post-transfection compared to the cells treated with TSA at the time of transfection. We also verified by qPCR the amount of luciferase gene in the HaCaT cells under different conditions and timing of TSA treatment after five days, without selection. The results shown in Figure $4 \mathrm{C}$ are in agreement with the results shown in Figure 4A and 4B, suggesting that TSA treatment improves maintenance of transfected DNA, in particular if TSA induction is performed post-transfection.

\section{TSA induces HaCaT cell differentiation that can be inhibited by the HPV-16 LCR presence at the time of treatment}

An important difference between HeLa, SiHa and $\mathrm{HaCaT}$ cell lines is that the first two are immortalized, while the latter can differentiate, providing a possible clue to the different HDACi time-dependent responses in these cell lines (Figure 3), as only TSA time-dependent response could be observed in the $\mathrm{HaCaT}$ cells. To investigate the potential effect of TSA on HaCaT differentiation, we analyzed the staining of these cells under various conditions using the differentiation marker $\beta$-catenin. As shown in Figure 5A, control cells, not transfected and non-TSA treated (NT, NI) or pUC18 transfected and non-
A

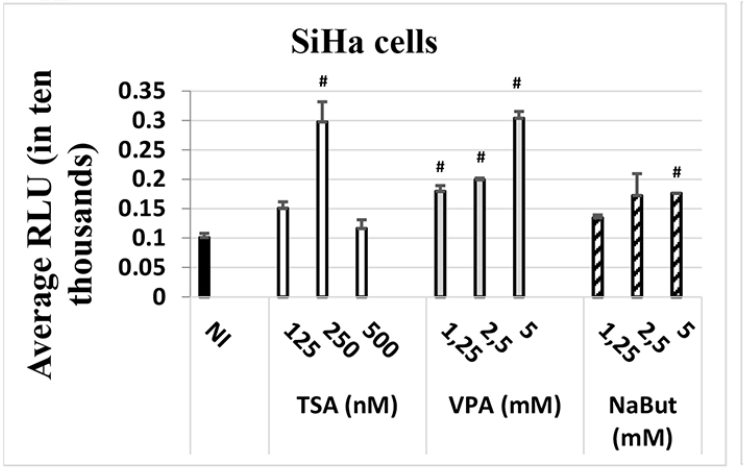

B

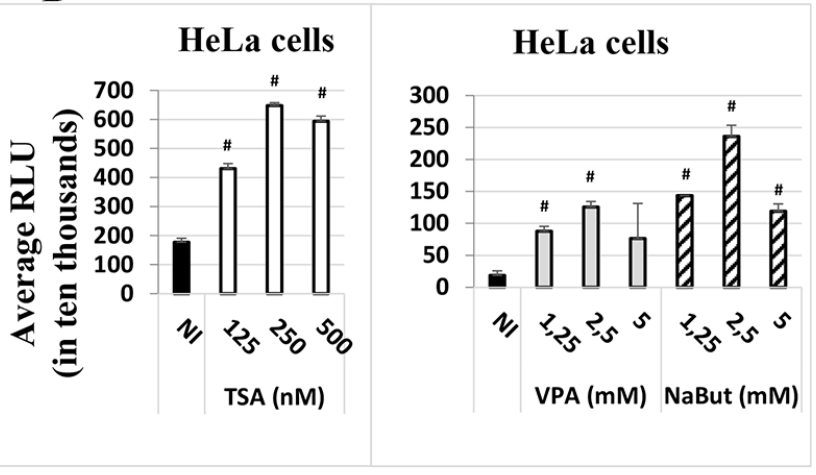

C

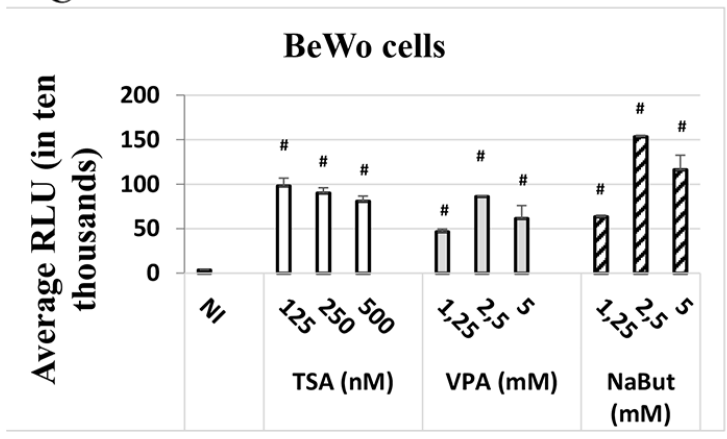

D

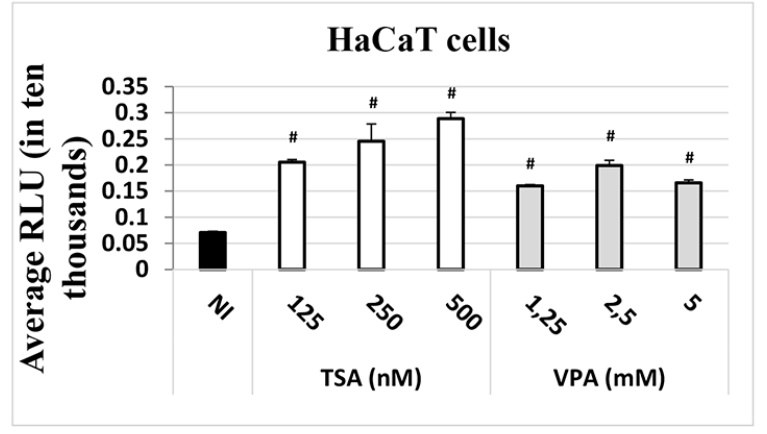

Figure 1: HDACi induce the expression of the luc gene under the control of the HPV-16 LCR in the SiHa. A. HeLa, B. BeWo C. and HaCaT D. cell lines. Cells were transfected with the HPV-16 pWtLCRluc plasmid. HDACi TSA (white), VPA (grey) or NaBut (striped) were added at the time of transfection at the concentrations indicated. NI: non-HDACi-treated transfected control cells (black). Twenty-four hours post-transfection, cells were lysed and intracellular protein luminescence (represented as average RLU units on the graphs) was read on a luminometer. The results are the average of at least three independent experiments for each cell line indicated. Bars represent standard deviations. Hash marks above standard deviations represent statistically significant data, compared to NI. 
TSA treated, are undifferentiated, according to a diffuse cytoplasmic staining of the $\beta$-catenin. HaCat transfection had thus no impact on the HaCat cell differentiation. In contrast, cells not transfected but treated with TSA at T0 or T6 or pUC18 transfected and treated with TSA at T0 (Figure 5A) and T6 (data not shown) or pWtLCRLuc transfected and treated with TSA at T6 (Figure 5B) show marks of differentiation, with a defined ("cage-like") $\beta$-catenin staining. This confirmed that TSA promotes the differentiation of keratinocytes, irrespective of the time of treatment or of transfection. However, in pWtLCRLuc transfected $\mathrm{HaCaT}$ cells, treated with TSA at T0, surprisingly, we observed no cell differentiation. From these data, we concluded that TSA tends to differentiate keratinocytes, that this effect is specifically reduced by the viral LCR (not observed in pUC18 transfected HaCaT cells) when it is introduced into the cells at the same time as the HDACi treatment and that LCR transcriptional activity is increased by TSA in undifferentiated $\mathrm{HaCaT}$ cells (T0) but decreased by TSA in differentiated HaCaT cells (T6) (Figures 3 and 5).

\section{The HPV-16 LCR transcriptional response to TSA treatment in $\mathrm{HaCaT}$ cells is dependent on AP-1 binding sites}

As the HPV-16 LCR contain many different binding sites for homo- or hetero-dimers transcription factors
A
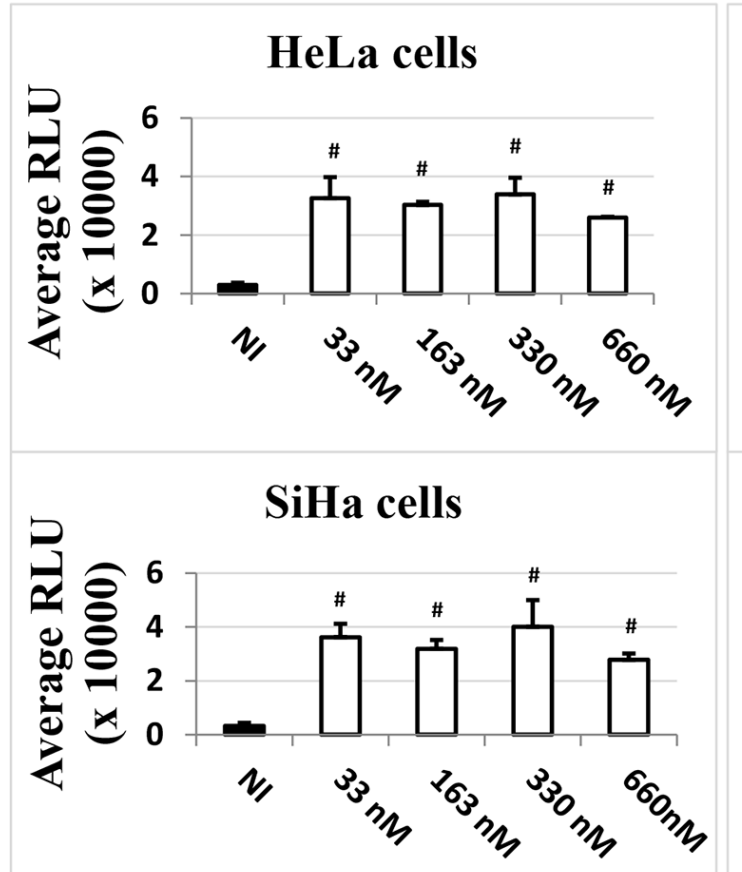

B
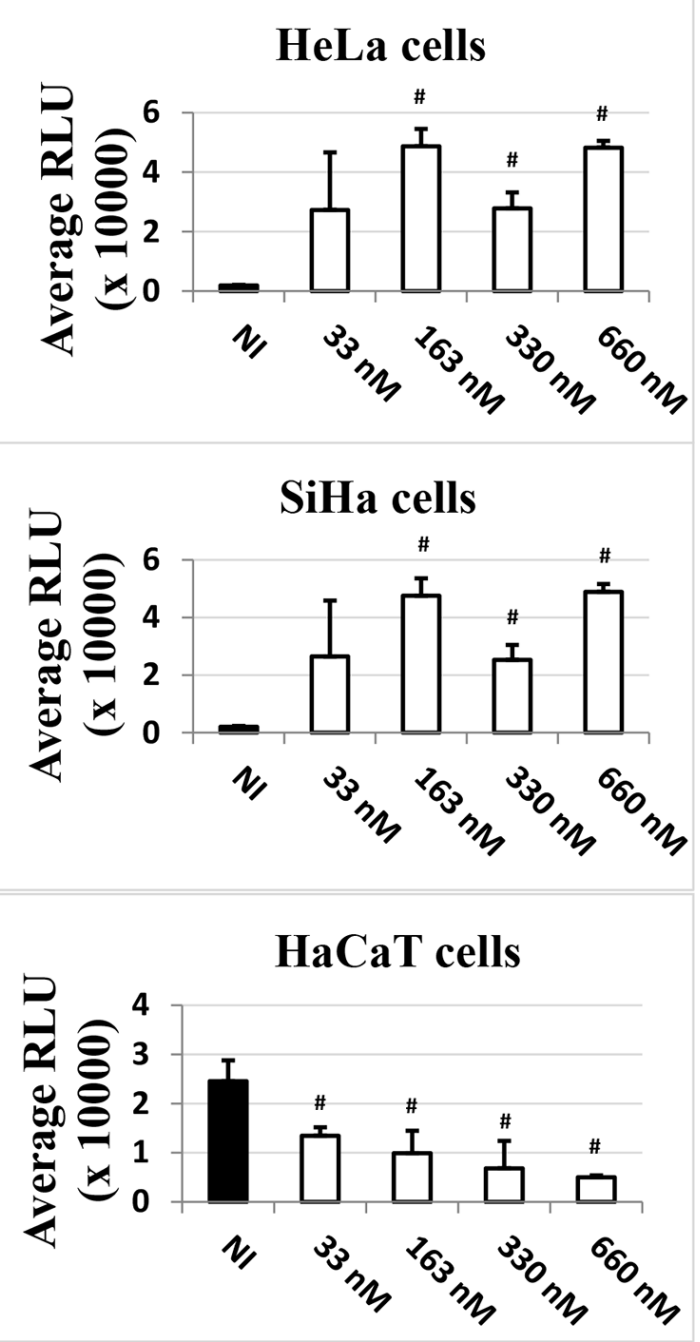

Figure 2: Time-dependent effect of TSA on the LCR transcriptional activity in HeLa, SiHa and HaCaT transformed cell lines. Cells were transfected with pLCRluc. TSA (white) was added in the indicated doses either at the time of transfection A. or six hours post-transfection B. pWtLCRluc-transfected, non TSA-induced (NI, black) cells were used as controls. Twenty-four hours post-transfection, cells were lysed and the luciferase activity was measured in terms of RLU units. These results are the average of three independent experiments done in triplicate. Bars indicate standard deviations. Hash marks above standard deviations indicate statistically significant data, compared to NI. 
(AP-1 and C/EBP $\beta$ transcription factors), we studied the involvement of the 3 major AP-1 binding sites of the HPV16 LCR on the observed TSA effect in transfected HaCat cells. For this purpose, we compared the luciferase activity in $\mathrm{HaCaT}$ cells transfected either with the wild type pWtLCRluc plasmid or with the mutated AP-1 derived pWtLCRluc plasmid, p4el4er9luc, in which all three major AP-1 binding sites in the LCR region of HPV-16 have been mutated [32]. In the absence of TSA treatment, we observed a marked luciferase activity decrease in cells transfected with the AP-1 mutant construct, consistent with the reported role of AP-1 in HPV LCR-driven transcription. Interestingly, addition of increasing doses of TSA (at T0 or T6) to the p4el4er9luc - transfected cells, failed to reproduce the effects observed on $\mathrm{HaCaT}$ cells transfected with its wild-type counterpart and treated with the same TSA doses (Figure 6). The three major AP-1 binding sites are thus required for the effects of TSA on the transcriptional activity of the HPV-16 LCR.

\section{DISCUSSION}

It is a general opinion in the scientific literature that HDACi could be used as potential drugs to cure cervical cancer. By virtue of their ability to induce differentiation, growth arrest and apoptosis in several cancer cell line models, these agents were introduced into clinical trials [29]. Prominent studies by Finzer et al. have shown that HDACi (e.g. NaBut, phenylbutyrate and TSA) can arrest cells at the $\mathrm{G} 1$ to $\mathrm{S}$ transition of the cell cycle in the HPV-positive HeLa cell line [19, 20]. Finzer et al. have also demonstrated that HDACi induce an intrinsic type
A
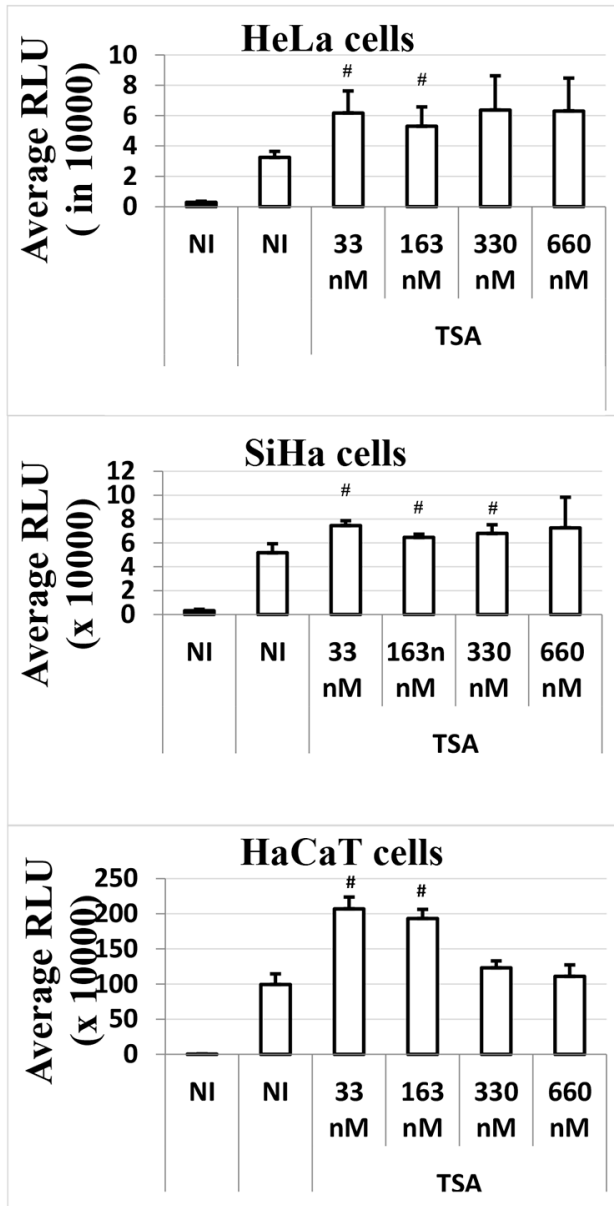

B
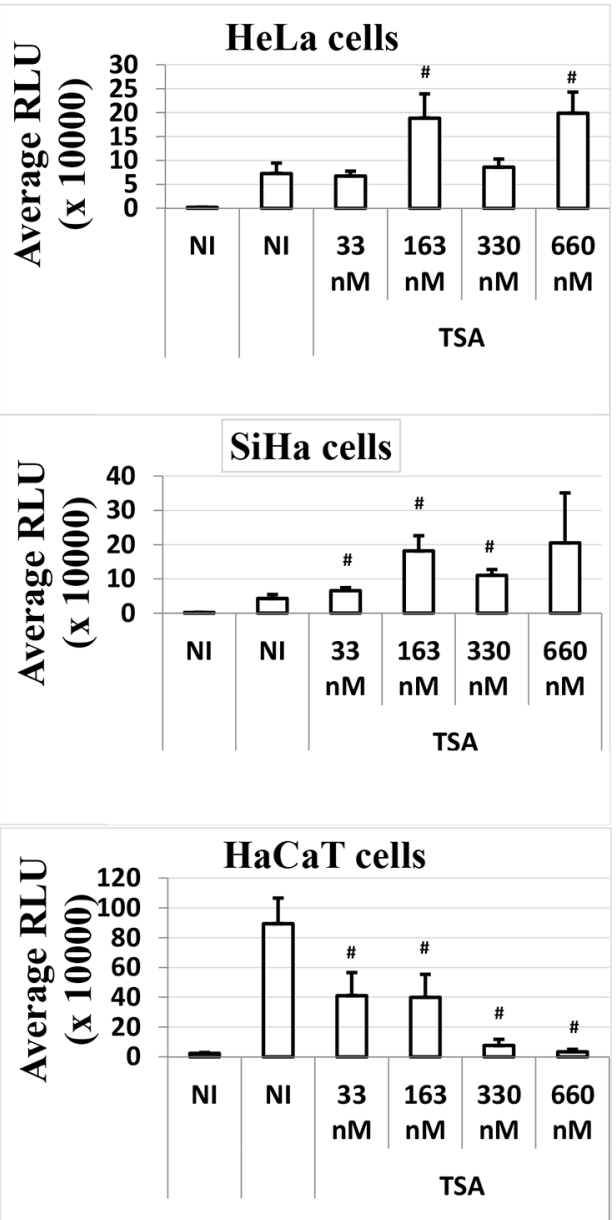

Figure 3: Time-dependent effect of TSA on the LCR transcriptional activity in the presence of HPV-16 early genes in HeLa, SiHa and HaCaT transformed cell lines. Cells were transfected as usual with the pWtLCRluc plasmid alone (NI, black), or transfected together with the pLCRearly plasmid (NI, white) and treated with TSA (white). TSA was added either at the time of transfection, T0 A. or six hours post-transfection, T6 B. Average RLUs were obtained from three independent experiments done in triplicate. Bars represent standard deviations. Hash marks above standard deviation represent statistically significant data, compared to NI. 
of apoptosis in HPV-positive cells [18]. In a subsequent study, Darvas et al. additionally showed that HDACi sensitize resistant HeLa cells to receptor-induced cell death [17]. Despite these insights, little is known about the impact of these agents in the context of the HPV life cycle and its outcome on HPV infection. This is particularly important, as it is known that most cervical lesions, although caused by infection with only one type of HR HPV, are typically surrounded by co-infections with multiple viruses $[33,34]$. In an effort to study these issues in more detail, we first evaluated the impact of several HDACi on the HPV-16 LCR-driven transcription in various cell lines. We demonstrated that TSA, VPA and $\mathrm{NaBut}$ induce several-fold the transcription of the firefly luciferase reporter gene under the control of the HPV$16 \mathrm{LCR}$ in the cervical cell lines HeLa and $\mathrm{SiHa}$, but also in the HPV-negative trophoblastic cell line BeWo. Furthermore, TSA and VPA induced a 2-4-fold increase in the LCR-driven transcription of the luciferase reporter in $\mathrm{HaCaT}$ cells, an HPV-negative keratinocyte cell line able to differentiate (unlike HeLa, SiHa and BeWo cells). This is in agreement with Bechtold et al. reporting that E6/ E7 mRNA production was upregulated by TSA from an episomal viral DNA in the W12 keratinocyte cell line [31]. The accessibility of the HPV-16 LCR to the numerous transcriptional HPV-16 LCR activators, able of crosscoupling, such as AP-1 and NF-IL6/C/EBP $\beta$, could be, as expected, improved $[35,36]$. Some of these transcriptional activators, for instance AP-1, has been reported to interact with HAT or HDAC and to regulate positively or negatively promoters $[37,38]$. We previously observed that c-jun protein can cooperate with $\mathrm{CBP} / \mathrm{p} 300$ general coactivators to activate the HPV-16 LCR [39] and Stiehl et al. reported that $\mathrm{CBP} / \mathrm{p} 300$, interacting with various transcription factors but also with regulatory proteins such as HAT, can be autoacetylated [40]. This autoacetylation

A
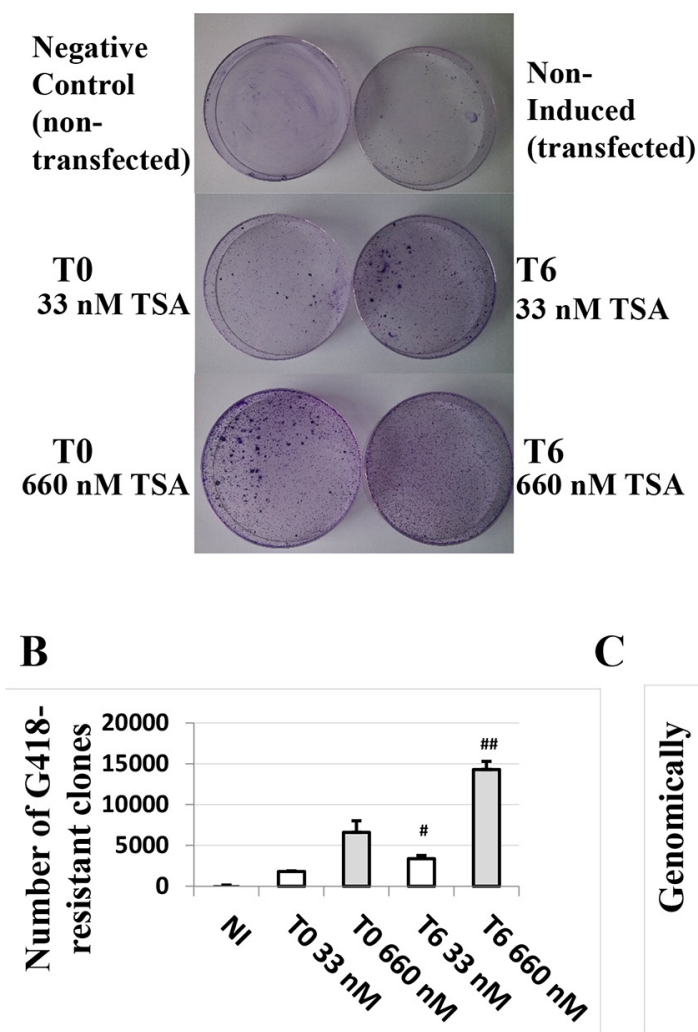

C

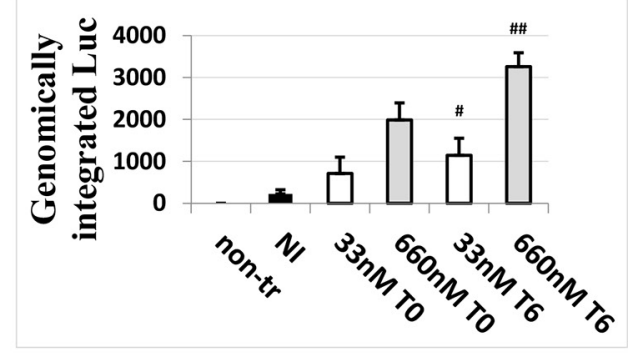

Figure 4: TSA treatment of cells transfected with the HPV-16 LCR causes a dose- and time of induction-dependent integration of the LCR-carrying episomal DNA into the genome of HaCaT cells. Cells were transfected with pWtLCRluc and treated with $33 \mathrm{nM}$ or $660 \mathrm{nM}$ TSA at T0 (the time of transfection) or T6 (six hours post-transfection). G 418-resistant clones were stained 10 days post-transfection A. and quantified B. The average numbers from three independent experiments are presented. Quantitative PCR for the luc gene was also performed on the genomic DNA of non-treated cells, transfected but non-induced cells (NI, black) and TSA-treated, transfected cells five days post-transfection in the absence of selection C. Presented are the average numbers from three independent experiments done in triplicate. Bars indicate standard deviations. Hash marks represent statistically significant data, comparing the T0 and T6 33 nM-TSA dose (white, single hash marks) and the T0 and T6 $660 \mathrm{nM}$-TSA dose (grey, double hash marks). 
can in turn regulate its own HAT activity and can be affected by TSA, increasing its dynamic transcriptional activity [40]. The HPV-16 LCR can also be activated by $\mathrm{C}$-EBP $\beta$ through a multiple $\mathrm{c} / \mathrm{EBP} \beta$ binding sites present in the LCR [39] and interestingly the transcriptional activity of this factor has also been reported to be activated by p300 acetylation [41]. So, it is tempting to think that the TSA treatment could have positively regulated the HPV-16 LCR activity through the activation of the p300/ CBP which in turn activated various transcription factors binding multiple binding sites.
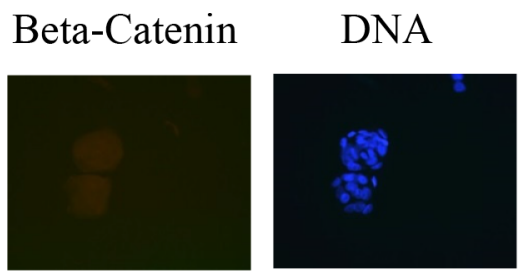

$\mathrm{NT}, \mathrm{NI}$
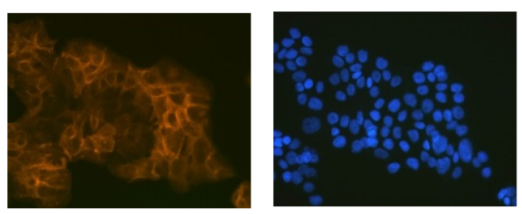

NT, I 660 nM TSA

T0
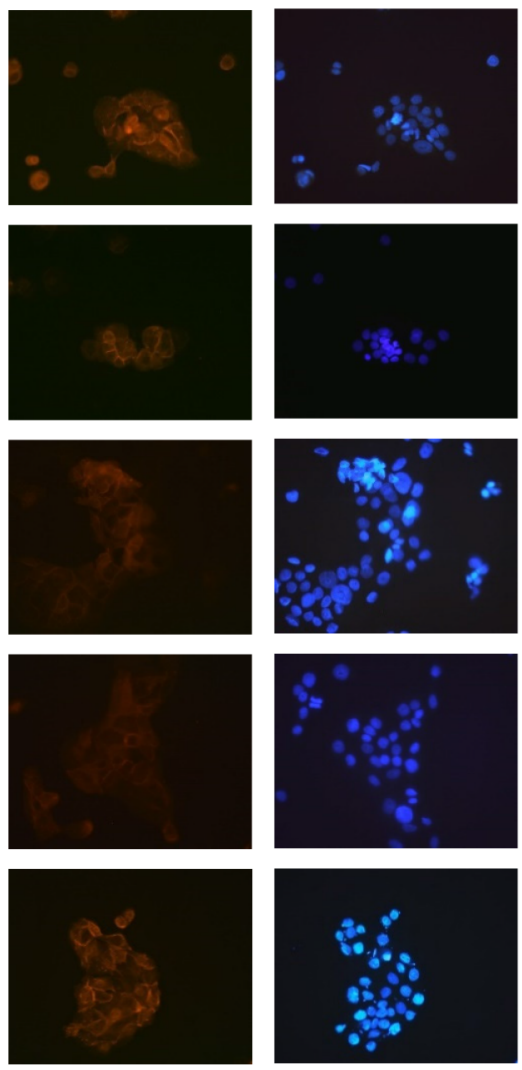

To assess the impact of HDACi on the transcriptional activity of LCR-controlled genes directly, excluding possible effects of these agents on other host cell and viral modifications - e.g. cell differentiation or viral DNA maintenance - or on the transfection efficiency, we evaluated the effect of adding TSA concurrently with transfection, or conversely, six hours post-transfection. According to our results, TSA had a clear "boosting" effect on the HPV-16 LCR-driven transcriptional activity in HeLa and $\mathrm{SiHa}$ cells, both when TSA treatment was performed at the time of transfection or post-transfection. The fact that
NT, I 660 nM TSA

T6

T, pUC, I 660 nM TSA

T0

T, NI,

pLCRluc

T, pLCRluc, I 660 nM TSA

T0

T, pLCRluc, I 660 nM TSA, T6

Figure 5: TSA induces the differentiation of keratinocytes, and this differentiation is overcome by the HPV-16 LCR. Cells were transfected with pWtLCRluc (T, pLCR, B) and treated with TSA (I) concomitantly with transfection (T0), or six hours later (T6). Non-transfected and non-induced (NT, NI), non-transfected and TSA- induced (NT, I), or cells transfected with pUC-18 (T, pUC, A) were used as controls. Cells were prepared for immuno-fluorescence and photographed. 
TSA could still strongly activate the LCR transcriptional activity even added 6 hours post-transfection alleviated the doubt that this activation could have been linked to transfection efficiency. However, surprisingly, although TSA increased the LCR transcriptional activity in HaCaT cells when added at T0, added six hours posttransfection it clearly reduced the LCR transcriptional activity, independently of HPV early gene expression. This suggested an additional effect of TSA in HaCaT cells on either the host cell or the transfected DNA - e.g. cell differentiation or DNA integration, as confirmed later. Indeed, HeLa cells and SiHa cells are immortalized, unable to differentiate but HaCaT cells treated with TSA showed marked signs of differentiation, notably when TSA was added 6 hours post-transfection (T6) and that it reduced the LCR transcriptional activity. This effect of TSA on the keratinocyte differentiation is in agreement with previous results from Markova et al. on cultured epidermal

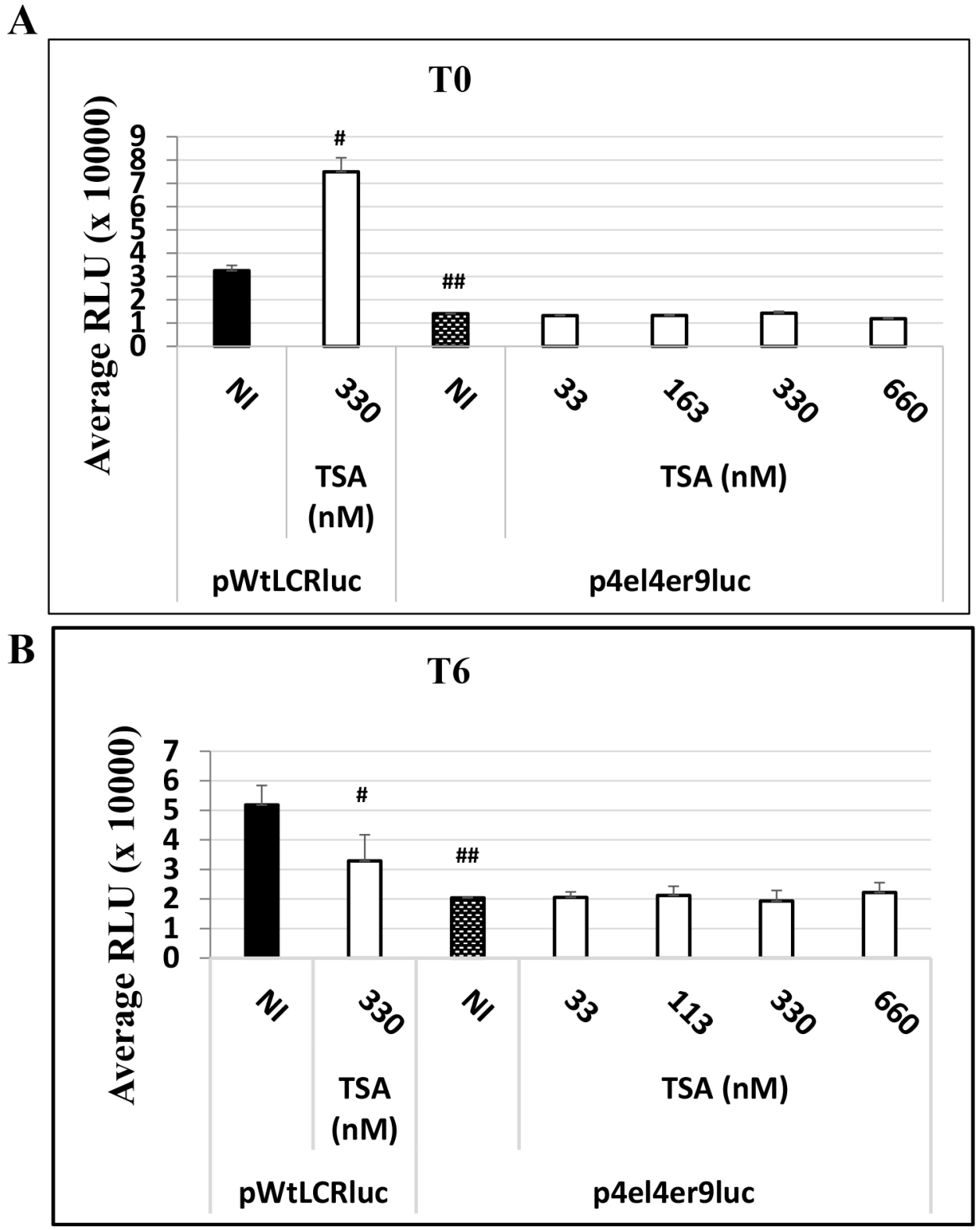

Figure 6: The HPV-16 LCR time-dependent transcriptional response to TSA treatment is dependent on AP-1 binding sites in HaCat cells. HaCaT cells were transfected either with the wild-type pWtLCRluc plasmid (NI, black) or with the AP-1 binding sites-mutated plasmid, p4el4er9luc, either untreated (NI, dotted) or treated with TSA at the time of transfection A. T0, white for various doses of TSA), or six hours post-transfection B. T6, white for various doses of TSA). Luciferase activity was scored in RLU units as usual. The results presented are the averages of three independent experiments done in triplicate. Bars indicate standard deviations. Hash marks above standard deviations represent statistically-significant data, compared to NI pWtLCRluc (black). 
explants treated with TSA [42] and the HDACi effects on differentiation was already documented on various cells $[43,44]$. It is worth noting that TSA-induced differentiation seemed specifically inhibited when pWtLCRLuc plasmid was transfected at the time of the TSA treatment (T0), when TSA is able to activate the LCR promoter, suggesting a signaling competition between the differentiation program and the LCR transcriptional activity. Indeed, the artificially high number of pWtLCRluc copies introduced into the $\mathrm{HaCaT}$ cells at T0 during transient transfection could "titrate away" some transcription factors required for $\mathrm{HaCaT}$ cell differentiation, while at $\mathrm{T} 6$ the amount of available plasmids could have already dropped, as expected from a transient transfection and as observed in our integration analysis by qPCR. Durst et al., have shown that HPV early gene expression is inversely related to the differentiation of the host keratinocyte [45]. Various differentiation-regulated transcriptional factors could be involved, as many of those (e.g. AP-1, YY-1, CDP/Cut, NF-IL-6/C-EBP $\beta$ ) can bind and regulate the HPV-16 LCR $[35,36,45-47]$. AP-1 and YY1 transcription factors have been shown to bind and regulate, positively or negatively, respectively, both the HPV-16 LCR but also genes promoters involved in differentiation [37, 47-50]. As Wang et al. showed that acetylation of dimeric AP-1 complexes enhanced their binding to HPV-16 DNA [35], hyperacetylated conditions in $\mathrm{HaCaT}$ cells during treatment of transfected cells with TSA at time zero, could for example preferentially promote the binding of transcriptionactivating AP-1 complexes such as Fra-2/AP-1 to the HPV16 LCR, which in turn would not be anymore "available" to bind and regulate promoters involved in the differentiation genes expression. This hypothesis was partly investigated comparing the effect of TSA on the luciferase activity of HaCat cells transfected either with the pWtLCRLuc plasmid or with a mutated plasmid lacking the 3 major AP-1 binding sites. The lack of LCR upregulation by TSA at T0 and LCR downregulation by TSA at T6 when using the AP-1 binding sites mutated construct confirmed that transcriptional factors able to interact with at least one of the three major AP-1 binding sites of the HPV-16 LCR are indeed involved in those regulations. It is worth noting that we could not detect any effect of TSA on the luciferase activity when $\mathrm{HaCaT}$ cells were transfected with a classic artificial pAP-1-Luc reporter plasmid (luciferase gene expression under the control of tandem AP-1 binding sites in front of a minimal promoter), suggesting that other element(s) in the HPV-16 LCR (1000 pb between nt 7007 and nt 117 of the viral genome) are also necessary (data not shown).

Another factor responsible for the temporal transcriptional response to TSA treatment in $\mathrm{HaCaT}$ cells could be linked to the physical state (extra-chromosomal/ integrated) of the pWtLCRluc transfected DNA. To our knowledge, this is the first study on the effect of TSA treatment on the integration efficiency of extra- chromosomal DNA. We show that, both in experiments studying stable transfection efficiency, as well as, in qPCR experiments, TSA treatment of $\mathrm{HaCaT}$ cells promotes an improved maintenance of transfected DNA in the cell. As we used a plasmid unable to replicate without viral early protein expression and as 5-10 days post-transfection, only integrated DNA can be maintained in the cells, our results clearly suggest that HDACi promoted extra-chromosomal DNA integration. HDACi are known to induce chromatin decondensation, increasing DNA exposure, but are also known to downregulate the activity of the DNA repair machinery [51-53]. It is therefore tempting to think that this might have also promoted the integration of extrachromosomal DNA. Considering that Bechtold et al., have shown that TSA boosts transcription of the E6 and E7 early genes from episomal templates (in agreement with our data), while suppressing it from intra-chromosomal viral templates [31], it is possible that the decrease in transcription efficiency of the HPV-16 LCR, promoted by TSA treatment post-transfection, could be partially due to the TSA-promoted increase of integrated copies of the pWtLCRluc in HaCaT cells. However, we think that the impact of TSA on HaCaT differentiation, is more important than the role of TSA on the integration DNA status in reducing LCR-driven transcription. In the HIV field, HDACi have also entered clinical trials [22]. While combinatory antiretroviral therapy (CART) is capable of durably suppressing viremia in HIV-infected individuals, it is unable to cure infection. The best understood barrier to eradicating HIV infection is the persistence of a latent reservoir of infected resting memory CD4+ T cells. By virtue of their quiescent state, these cells are not thought to express HIV-1 antigens, rendering them insensitive to CART and able to escape the host immune system. HDACi are well-known inducers of transcription from the HIV-1 promoter [22, 26-28]. As such, these drugs are currently tested in combination with CART for their abilities to induce viral expression in the resting CD4+ T-cell reservoir in order to trigger immune-mediated clearance of infected cells. Given that both the HIV-1 and HPV promoters are regulated by similar transcription factors (e.g., C/ЕBP $\beta$, AP-1, NF-кB, YY-1) [35, 36, 49, 50, 54-58], it is not surprising that transcription from both viral promoters can be induced by HDAC inhibition, unless, as shown in our study, HDACi promote differentiation and/or viral DNA integration. In conclusion, our results suggest that HDACi could affect the integration of viral extra-chromosomal DNA into the genome of the host cells, but also engender a dangerous episomal burst of transcription from the HPV viral promoter in undifferentiated cells. The molecular mechanisms elucidating these events will be the subject of further studies. Our results therefore underscore the need to carry out further experiments, especially in vivo in mouse models or with clinical samples, to better evaluate the impact of the use of HDACi in the treatment of HPV infection. 


\section{MATERIALS AND METHODS}

\section{Plasmids}

The construction of the pLCR early (or pHPV16 early) plasmid containing the HPV16 early genes under the control of the HPV16 LCR cloned in the pCR-XLTOPO vector, has been previously described [32]. The pWtLCRluc plasmid containing the HPV16 LCR in front of the firefly luciferase gene in pUHC 13.3 was also previously described [54]. The AP-1 binding sites mutant plasmid, p4el4el9luc, was obtained by site-directed mutagenesis of pWtLCRluc, [32]. The peGFP-NLS was a kind gift of X. Saelens (Molecular Virology, VIB Department for Molecular Biomedical Research, Ghent University, Belgium) and the pAP-1-Luc was a kind gift of Y. Jacob (Virus interactomics, Institut Pasteur de Paris, France).

\section{Cell culture, transfections and drug treatment}

The trophoblastic BeWo cell line, keratinocyte $\mathrm{HaCaT}$ cell line, and HeLa (HPV-18-harbouring) and $\mathrm{SiHa}$ (HPV-16-harbouring) immortalized cervical epithelium cell lines were maintained in DMEM (Gibco) supplemented with $10 \%$ fetal calf serum (Gibco), 1\% penicillin/streptomycin and 1\% Non-Essential Amino Acids (Lonza). Transfections of HeLa and SiHa cells were carried out by the calcium phosphate precipitation method, as described previously [54, 59]. Transfections of BeWo cells were done using the jetPEI transfection reagent (Polyplus-transfection Inc), according to the manufacturer's specifications. HaCaT cells were transfected using the ViaFect transfection reagent (Promega), again according to the manufacturer's specifications. The HDACi Valproate (VPA), sodium butyrate (NaBut) and trichostatin A (TSA) were a kind gift from Dr. Carine van Lint (ULB). Serial dilutions in serumfree DMEM were used to prepare fresh aliquots of each inhibitor at the desired range of concentrations and cells were treated at the time of transfection (T0) or six hours post-transfection (T6), as required. Parallel transfection using the peGFP-NLS reporter plasmid allowed us to assess the transfection efficiency in our experiments by direct fluorescent cell visualization under the fluorescence microscope. A transfection efficiency of $40-60 \%$ was routinely achieved.

\section{Measurement of luciferase activity}

Cells were transfected and HDACi-treated as described above. To assess for transfection efficiency, cells were transfected in parallel with peGFP-NLS. A transfection efficiency of $40 \%$ to $60 \%$ (assessed by visual inspection of the cells under the fluorescent microscope) was routinely achieved. Luciferase activity was measured 24 hours post-transfection using the Luciferase Assay System (Promega). Briefly, $100 \mu \mathrm{L}$ Luciferase Assay Reagent was injected into $20 \mu \mathrm{L}$ cell lysate per transfected sample. Relative Luminescence Units (RLU) were measured for 60 s using a luminometer (Berthold Technologies, Germany). Luciferase activity was normalized to the total protein content of the cell lysates, as determined by the Bradford reagent (Sigma-Aldrich). The results shown are representative of at least three different experiments using different DNA preparations.

\section{Assessment of genomic integration of pWtLCRluc: G418 selection and qPCR}

HaCaT cells were transfected with pWtLCRluc together with pCMVeGFP (carrying the Neomycinresistance gene; ratio 10:1) and HDACi-treated in six-well plates. Twenty-four hours post-transfection, 50,000 cells per each condition were transferred into $10 \mathrm{~cm}$-plates and further incubated with G-418 to a final concentration of $500 \mu \mathrm{g} / \mathrm{mL}$. Selection continued for 10 days, with addition of fresh G-418-containing medium every 2-3 days. At the end of the selection period, cells were washed with PBS, fixed with $100 \%$ ice-cold methanol, and stained with Crystal violet. G-418-resistant clones were photographed and counted on a bacterial cell counter. For the qPCR, five days post-transfection with $\mathrm{pWtLCRluc}$, cells were washed with the CellScrub washing buffer (Genlantis) in order to remove cationic lipid/DNA complexes associated with the cell surface after transfection. Total DNA was extracted using the DNA Realeasy reagent (Nippon Genetics), according to the manufacturer's specifications. To discriminate between integrated and input episomal pWtLCRluc copies, and to normalize for transfection efficiency, isolated DNA was digested with DpnI (digests dam methylated bacterial DNA). Samples were purified with phenol-chloroform-isoamyl alcohol (25:24:1), ethanol-precipitated, washed with $70 \%$ ethanol, dried and resuspended in TE. The primers used to amplify the firefly luciferase reporter gene have already been described [32]. qPCR was performed using the IQ SYBR Green Supermix (BioRad), on the BioRad Thermal Cycler, under the following conditions: a DNA polymerase activating step at $95^{\circ} \mathrm{C}$ for 3 minutes, followed by 40 cycles of denaturation at $95^{\circ} \mathrm{C}$ for 1 minute and annealing/elongation at $60^{\circ} \mathrm{C}$ for 30 seconds. As reference genes, $\beta$ Actin and GAPDH were also amplified simultaneously with the firefly luciferase gene, and the relative copy number of genomically integrated pWtLCRluc was calculated by the $\Delta \Delta \mathrm{Ct}$ method. The primers for amplifying the two reference genes have already been described [32].

\section{Immunolabelling for fluorescence microscopy}

$\mathrm{HaCaT}$ cells were plated on 8-well slides and transfected and HDACi-treated as described already. At 
24 hours post-transfection, cell preparations were rinsed with PBS and fixed with 4\% paraformaldehyde (PAF) for $20 \mathrm{~min}$. After several more washes with PBS and in $\mathrm{PBS} / 0.01 \%$ triton $\mathrm{X}-100$, non-specific sites on cells were blocked with a 20-times dilution of normal donkey antibody and the slides were left at room temperature for 1 hour. After this, a 100-fold dilution of mouse anti$\beta$-catenin antibody (Santa Cruz Biotechnologies) was applied to the cells and slides were left to incubate in a humid chamber for 24 hours at $4{ }^{\circ} \mathrm{C}$. All further steps were carried out in the dark. Namely, after two washes in PBS, cell preparations were incubated in a 100 -fold dilution of donkey-anti-mouse $\mathrm{Cy} 3$ (Vector laboratories) secondary antibody, for 30 minutes at room temperature. After that, washed labelled cells were mounted with Prolong Gold antifade reagent with DAPI (Invitrogen). A week later, dried slides were visualized by fluorescence microscopy.

\section{Statistics}

The data presented are representative of at least three independent experiments done in triplicate using different DNA preparations. Statistical analyses were performed using MS Office software. Statistical differences were determined by using a two-tailed, unpaired Students' t-test. A $p$ value of less than 0.05 was considered as statistically significant.

\section{CONFLICTS OF INTEREST}

The authors declare no conflicts of interest.

\section{REFERENCES}

1. Antonsson A, Forslund O, Ekberg H, Sterner G, Hansson BG. The ubiquity and impressive genomic diversity of human skin papillomaviruses suggest a commensalic nature of these viruses. J Virol. 2000; 74:11636-41.

2. Antonsson A, Erfurt C, Hazard K, Holmgren V, Simon M, Kataoka A, Hossain S, Håkangård C, Hansson BG. Prevalence and type spectrum of human papillomaviruses in healthy skin samples collected in three continents. J Gen Virol. 2003; 84:1881-6.

3. zur Hausen H. Papillomaviruses in the causation of human cancers - a brief historical account. Virology. 2009; 384:260-265.

4. Herfs M, Yamamoto Y, Laury A, Wang X, Nucci MR, McLaughlin-Drubin ME, Münger $\mathrm{K}$, Feldman $\mathrm{S}$, McKeon FD, Xian W, Crum CP. A discrete population of squamocolumnar junction cells implicated in the pathogenesis of cervical cancer. Proc Natl Acad Sci USA. 2012; 109:10516-10521.

5. Mirkovic J, Howitt BE, Roncarati P, Demoulin S, Suarez-Carmona M, Hubert P, McKeon FD, Xian W, Li A, Delvenne P, Crum CP, Herfs M. Carcinogenic HPV infection in the cervical squamo-columnar junction. J Pathol. 2015; 236:265-271.

6. Moody CA, Laimins LA. Human papillomavirus oncoproteins: pathways to transformation. Nat Rev Cancer. 2010; 10:550-560.

7. Thomas JT, Hubert WG, Ruesch MN, Laimonis LA. Human papillomavirus type 31 oncoproteins E6 and E7 are required for the maintenance of episomes during the viral life cycle in normal human keratinocytes. Proc Natl Acad Sci USA. 1999; 96:8449-8454.

8. Park RB, Androphy EJ. Genetic analysis of high-risk E6 in episomal maintenance of human papillomavirus genomes in primary human keratinocytes. J Virol. 2002; 76:11359-11364.

9. Sénéchal H, Poirier GG, Coulombe B, Laimins LA, Archambault J. Amino acid substitutions that specifically impair the transcriptional activity of papillomavirus E2 affect binding to the long isoform of Brd4. Virology. 2007; 358:10-7.

10. Jang MK, Shen K, McBride AA. Papillomavirus genomes associate with BRD4 to replicate at fragile sites in the host genome. PLoS Pathog. 2014; 10:e1004117.

11. Timmermann S, Lehrmann H, Polesskaya A, Harel-Bellan A. Histone acetylation and disease. Cell Mol Life Sci. 2001; 58:728-736.

12. Yoshida M, Horinouchi S, Beppu T. Trichostatin A and trapoxin: novel chemical probes for the role of histone acetylation in chromatin structure and function. Bioessays. $1995 ; 17: 423-430$.

13. Mitsiades N, Mitsiades CS, Richardson PG, McMullan C, Poulaki V, Fanourakis G, Schlossman R, Chauhan D, Munshi NC, Hideshima T, Richon VM, Marks PA, Anderson KC. Molecular sequelae of histone deacetylase inhibition in human malignant B cells. Blood. 2003; 101:4055-4062.

14. Wang R, Brunner T, Zhang I, Shi Y. Fungal metabolite RF901228 inhibits c-Myc and Fas ligand expression. Oncogene. 1998; 17:1503-1508.

15. Arts J, Lansink M, Grimbergen J, Toet KH, Kooistra T. Stimulation of tissue-type plasminogen activator gene expression by sodium butyrate and trichostatin A in human endothelial cells involves histone acetylation. Biochem J. 1995; 310:171-176.

16. Choi JH, Nam KH, Kim J, Baek MW, Park JE, Park HY, Kwon HJ, Kwon OS, Kim DY, Oh GT. Trichostatin A exacerbates atherosclerosis in low density lipoprotein receptor-deficient mice. Arterioscler Thromb Vasc Biol. 2005; 25:2404-9.

17. Darvas K, Rosenberger S, Brenner D, Fritsch C, Gmelin N, Krammer PH, Rösl F. Histone deacetylase inhibitorinduced sensitization to TNFalpha/TRAIL-mediated apoptosis in cervical carcinoma cells is dependent on HPV oncogene expression. Int $\mathrm{J}$ of Cancer. 2010; 127:1384-1392. 
18. Finzer P, Krueger A, Stöhr M, Brenner D, Soto U, Kuntzen C, Krammer PH, Rösl F. HDAC inhibitors trigger apoptosis in HPV-positive cells by inducing the E2F-p73 pathway. Oncogene. 2004; 23:4807-17.

19. Finzer P, Stöhr M, Seibert N, Rösl F. Phenylbutyrate inhibits growth of cervical carcinoma cells independent of HPV type and copy number. J Cancer Res Clin Oncol. 2003; 129:107-13.

20. Finzer P, Kuntzen C, Soto U, zur Hausen H, Rösl F. Inhibitors of histone deacetylase arrest cell cycle and induce apoptosis in cervical carcinoma cells circumventing human papillomavirus oncogene expression. Oncogene. 2001; 20:4768-76.

21. Wollebo HS, Bellizzi A, Cossari DH, Safak M, Khalili K, White MK. Epigenetic regulation of polyomavirus JC involves acetylation of specific lysine residues in NF- $\mathrm{KB}$ p65. J Neurovirol. 2015; 21:679-87.

22. Demonté D, Quivy V, Colette Y, Van Lint C. (2004) Administration of HDAC inhibitors to reactivate HIV-1 expression in latent cellular reservoirs: implications for the development of therapeutic strategies. Biochem Pharmacol; 68: 1231-1238.

23. Murata T, Kondo Y, Sugimoto A, Kawashima D, Saito $\mathrm{S}$, Isomura H, Kanda T, Tsurumi T. Epigenetic histone modification of Epstein-Barr virus BZLF1 promoter during latency and reactivation in Raji cells. J Virol. 2012; 86:4752-61.

24. Zhao W, Noya F, Chen WY, Townes TM, Chow LT, Broker TR. Trichostatin A up-regulates Human Papillomavirus Type 11 Upper Regulatory Region-E6 Promoter Activity in Undifferentiated Primary Human Keratinocytes. J Virol. 1999; 73: 5026-5033.

25. Shin HJ, DeCotiis J, Giron M, Palmeri D, Lukac DM. Histone deacetylase classes I and II regulate Kaposi's sarcoma-associated herpesvirus reactivation. J Virol. 2014; 88:1281-92.

26. Archin NM, Liberty AL, Kashuba AD, Choudhary SK, Kuruc JD, Crooks AM, Parker DC, Anderson EM, Kearney MF, Strain MC, Richman DD,Hudgens MG, Bosch $\mathrm{RJ}$, et al. Administration of vorinostat disrupts HIV-1 latency in patients on antiretroviral therapy. Nature. 2012; 487:482-485.

27. Archin NM, Espeseth A, Parker D, Cheema M, Hazuda D, Margolis DM. Expression of latent HIV induced by the potent HDAC inhibitor suberoylanilide hydroxamic acid. AIDS Res Hum Retroviruses. 2009; 25:207-212.

28. Rasmussen TA, Schmeltz Søgaard O, Brinkmann C, Wightman F, Lewin SR, Melchjorsen J, Dinarello C, Østergaard L, Tolstrup M. Comparison of HDAC inhibitors in clinical development: effect on HIV production in latently infected cells and T-cell activation. Hum Vaccin Immunother. 2013; 9:993-1001.

29. Minucci S, Pelicci PG. Histone deacetylase inhibitors and the promise of epigenetic (and more) treatments for cancer. Nat Rev Cancer. 2006; 6:38-51.
30. Stünkel W, Bernard HU. The chromatin structure of the long control region of human papillomavirus type 16 represses viral oncoprotein expression. J Virol. 1999;73:1918-30.

31. Bechtold V, Beard P, Raj K. Human papillomavirus type $16 \mathrm{E} 2$ protein has no effect on transcription from episomal viral DNA. J Virol. 2003; 77: 2021-8.

32. Weyn C, Vanderwinden JM, Rasschaert J, Englert Y, Fontaine V. Regulation of human papillomavirus type 16 early gene expression in trophoblastic and cervical cells. Virology. 2011; 412:146-55.

33. van der Marel J, Berkhof J, Ordi J, Torné A, Del Pino M, van Baars R, Schiffman M, Wentzensen N, Jenkins D, Quint WG Attributing oncogenic human papillomavirus genotypes to high-grade cervical neoplasia: which type causes the lesion? Am J Surg Pathol. 2015; 39:496-504.

34. Schmitt M, Depuydt C, Benoy I, Bogers J, Antoine J, Arbyn M, Pawlita M. Multiple human papillomavirus infections with high viral loads are associated with cervical lesions but do not differentiate grades of cervical abnormalities. J Clin Microbiol. 2013; 51:1458-64.

35. Wang WM, Wu SY, Lee AY, Chiang CM. Binding site specificity and factor redundancy in activator protein1-driven human papillomavirus chromatin-dependent transcription. J Biol Chem. 2011; 286:40974-86.

36. Struyk L, van der Meijden E, Minnaar R, Fontaine V, Meijer I, ter Schegget J. Transcriptional regulation of human papillomavirus type $16 \mathrm{LCR}$ by different C/EBPbeta isoforms. J Mol Carcinog. 2000; 28:42-50.

37. Mittelstadt ML, Patel RC. AP-1 mediated transcriptional repression of matrix metalloproteinase- 9 by recruitment of histone deacetylase 1 in response to interferon $\beta$. PLoS One. 2012;7:e42152.

38. Rahman I, Gilmour PS, Jimenez LA, MacNee W. Oxidative stress and TNF-alpha induce histone acetylation and NF-kappaB/AP-1 activation in alveolar epithelial cells: potential mechanism in gene transcription in lung inflammation. Mol Cell Biochem. 2002; 234-235:239-48.

39. Fontaine V, van der Meijden E, ter Schegget J. Inhibition of human papillomavirus-16 long control region activity by interferon-gamma overcome by $\mathrm{p} 300$ overexpression. J Mol Carcinog. 2001; 31:27-36.

40. Stiehl DP, Fath DM, Liang D, Jiang Y, Sang N. Histone deacetylase inhibitors synergize p300 autoacetylation that regulates its transactivation activity and complex formation. Cancer Res. 2007; 67:2256-64.

41. Zhang J, Li Y, Shan K, Wang L, Qiu W, Lu Y, Zhao D, Zhu G, He F, Wang Y. Sublytic C5b-9 induces IL-6 and TGF$\beta 1$ production by glomerular mesangial cells in rat Thy-1 nephritis through p300-mediated C/EBP $\beta$ acetylation. FASEB J. 2014;28:1511-25.

42. Markova NG, Karaman-Jurukovska N, Pinkas-Sarafova A, Marekov LN, Simon M. Inhibition of histone deacetylation promotes abnormal epidermal differentiation and specifically suppresses the expression of the late 
differentiation marker profilaggrin. J Invest Dermatol. 2007; 127:1126-39.

43. Augeron C, Laboisse C. Emergence of permanently differentiated cell clones in a human colonic cancer cell line in culture after treatment with sodium butyrate. Cancer Res. 1984; 44:3961-3969.

44. Barnard JA, Warwick G. Butyrate rapidly induces growth inhibition and differentiation in HT-29 cells. Cell Growth Diff. 1993; 4:495-501.

45. Dürst M, Bosch FX, Glitz D, Schneider A, zur Hausen H. Inverse relationship between human papillomavirus (HPV) type 16 early gene expression and cell differentiation in nude mouse epithelial cysts and tumors induced by HPVpositive human cell lines. J Virol. 1991; 65:796-804.

46. O'Connor MJ, Stünkel W, Koh $\mathrm{CH}$, Zimmermann $\mathrm{H}$, Bernard HU. The differentiation-specific factor CDP/ Cut represses transcription and replication of human papillomaviruses through a conserved silencing element. J Virol. 2000; 74:401-10.

47. Tan SH, Baker CC, Stünkel W, Bernard HU. A transcriptional initiator overlaps with a conserved YY1 binding site in the long control region of human papillomavirus type 16. Virology. 2003; 20;305:486-501.

48. Dong XP, Stubenrauch F, Beyer-Finkler E, Pfister H. Prevalence of deletions of YY1-binding sites in episomal HPV 16 DNA from cervical cancers. Int J Cancer. 1994; 58:803-8.

49. Wurm S, Zhang J, Guinea-Viniegra J, García F, Muñoz J, Bakiri L, Ezhkova E, Wagner EF. Terminal epidermal differentiation is regulated by the interaction of Fra-2/AP-1 with Ezh2 and ERK1/2. Genes Dev. 2015; 29:144-56.

50. Taguchi S, Kawachi Y, Ishitsuka Y, Fujisawa Y, Furuta J, Nakamura Y, Xu X, Ikebe D, Kato M, Otsuka F. Overexpression of the transcription factor Yin-Yang-1 suppresses differentiation of $\mathrm{HaCaT}$ cells in threedimensional cell culture. J Invest Dermatol. 2011; 131:37-45

51. Marchion DC, Bicaku E, Daud AI, Sullivan DM, Munster PN. Valproic acid alters chromatin structure by regulation of chromatin modulation proteins. 2005; 65:3815-22.
52. Eot-Houllier G, Fulcrand G, Magnaghi-Jaulin L, Jaulin C. Histone deacetylase inhibitors and genomic instability. Cancer Lett. 2009; 18;274:169-76.

53. Thurn KT, Thomas S, Raha P, Qureshi I, Munster PN. Histone deacetylase regulation of ATM-mediated DNA damage signaling. Mol Cancer Ther. 2013; 12:2078-87.

54. Fontaine V, van der Meijden E, de Graaf J, ter Schegget J, Struyk L. A functional NF-kappaB binding site in the human papillomavirus type 16 long control region. Virology. 2000; 272:40-9.

55. Dahiya S, Liu Y, Nonnemacher MR, Dampier W, Wigdahl B. CCAAT enhancer binding protein and nuclear factor of activated T cells regulate HIV-1 LTR via a novel conserved downstream site in cells of the monocyte-macrophage lineage. PLoS One. 2014; 9:e88116.

56. Duverger A, Wolschendorf F, Zhang M, Wagner F, Hatcher B, Jones J, Cron RQ, van der Sluis RM, Jeeninga RE, Berkhout B, Kutsch O. An AP-1 binding site in the enhancer/core element of the HIV-1 promoter controls the ability ofHIV-1 to establish latent infection. J Virol. 2013; 87:2264-77.

57. Quivy V, Adam E, Collette Y, Demonte D, Chariot A, Vanhulle C, Berkhout B, Castellano R, de Launoit Y, Burny A, Piette J, Bours V, Van Lint C. Synergistic activation of human immunodeficiency virus type 1 promoter activity by NF-kappaB and inhibitors of deacetylases: potential perspectives for the development of therapeutic strategies. J Virol. 2002; 76:11091-103.

58. Coull JJ, Romerio F, Sun JM, Volker JL, Galvin KM, Davie JR, Shi Y, Hansen U, Margolis DM. The human factors YY1 and LSF repress the human immunodeficiency virus type 1 long terminal repeat via recruitment of histone deacetylase 1.J Virol. 2000; 74:6790-9.

59. van der Eb AJ, Graham FL. Assay of transforming activity of tumor virus DNA. Methods Enzymol. 1980; 65:826-39. 